\title{
Semi-Implicit Difference Scheme for a Two-Dimensional Parabolic Equation with an Integral Boundary Condition*
}

\section{Kristina Jakubėlienè $\dot{e}^{a}$, Regimantas Čiupaila ${ }^{b}$ and Mifodijus Sapagovas ${ }^{c}$}

\author{
${ }^{a}$ Kaunas University of Technology \\ Studentu̧ str. 50, LT-51368 Kaunas, Lithuania \\ ${ }^{b}$ Vilnius Gediminas Technical University \\ Saulètekio av. 11, LT-10223 Vilnius, Lithuania \\ ${ }^{c}$ Institute of Mathematics and Informatics, Vilnius University \\ Akademijos str. 4, LT-08663 Vilnius, Lithuania \\ E-mail(corresp.): kristina.jakubeliene@ktu.1t \\ E-mail: regimantas.ciupaila@vgtu.lt \\ E-mail: mifodijus.sapagovas@mii.vu.lt
}

Received July 5, 2016; revised June 10, 2017; published online September 15, 2017

\begin{abstract}
In this paper, we consider a finite difference method for a class of twodimensional parabolic equations with integral boundary conditions. The semi-implicit difference scheme is considered. The stability of difference scheme is proved using the properties of the $M$-matrices, particularly, the regular splitting of an $M$-matrix. The numerical results of some examples are presented, that approve our theoretical investigations.
\end{abstract}

Keywords: integral boundary condition, semi-implicit difference scheme, stability, $M$ matrix.

AMS Subject Classification: 65M06; 65M12; 65N25.

\section{Introduction}

The aim of the paper is to analyze a difference method for solving a twodimensional equation in a rectangular domain with an integral boundary condition. We construct a difference scheme, in which a nonlocal condition is taken on a lower layer. Then, on every layer we solve the difference problem with the Dirichlet condition. Our other objects are to analyze the stability of finite difference scheme by using some properties of $M$-matrices.

* The research was partially supported by the Research Council of Lithuania (grant No. MIP-047/2014). 
In the domain $Q_{T}=\Omega \times[0, T], \Omega=\{0<x, y<1\}$, we consider a linear two-dimensional parabolic equation

$$
\frac{\partial u}{\partial t}=\frac{\partial}{\partial x}\left(k(x, y) \frac{\partial u}{\partial x}\right)+\frac{\partial}{\partial y}\left(k(x, y) \frac{\partial u}{\partial y}\right)-q(x, y) u+f(x, y, t)
$$

with an integral boundary condition and initial condition

$$
\begin{aligned}
& u(x, y, t)=\iint_{\Omega} K(x, y, \xi, \eta) u(\xi, \eta, t) d \xi d \eta+\mu(x, y, t), \quad x, y \in \partial \Omega, \\
& u(x, y, 0)=\varphi(x, y), \quad x, y \in \Omega .
\end{aligned}
$$

A specific feature of this differential problem is that, in the nonlocal condition, the values of the solution at contour points are associated with the integral in the whole domain.

Numerical methods for parabolic equations with the different types of nonlocal conditions have been considered in many works. In a two-dimensional case one of the first articles in this direction was [5]. Later on, numerical methods for such a problem were analyzed in the articles $[1,7,15,18,22]$. Particularly, the two-dimensional problems with nonlocal conditions using one-dimensional difference schemes were investigated in $[6,10,20,25,31,32]$.

Fully implicit and semi-implicit difference schemes for problem (1.1)-(1.3) in the case $k=1, q=f=\mu=0$ were considered in paper [22]. It has been proven that, under some limitations for the kernel $K(x, y, \xi, \eta)$, both of these schemes are stable and the method of finite difference converges. In the case of both schemes, the differential equation is approximated by the same twolevel implicit scheme and both schemes differ one from the other only by the approximation of the integral boundary condition.

Condition (1.2) for a fully-implicit scheme is replaced by the condition

$$
u_{i j}^{n}=K_{i j}\left(\left\{u_{m l}^{n}\right\}\right),
$$

where

$$
K_{i j}\left(\left\{u_{m l}^{n}\right\}\right)=\sum_{m, l=0}^{N} \omega_{m l} K\left(x_{i}, y_{j}, x_{m}, y_{l}\right) u_{m l}^{n},
$$

indexes $(i, j)$ denote a point on the contour and $(m, l)$ is any internal or contour point, $\omega_{m l}$ are weights of the numerical integration formula.

The integral condition in the semi-implicit scheme is replaced by the explicit formula

$$
u_{i j}^{n}=K_{i j}\left(\left\{u_{m l}^{n-1}\right\}\right) .
$$

We notice that in all later published papers the theory of the finite difference method for the parabolic differential equations with various types of nonlocal conditions was created as fully-implicit or explicit schemes theory. In the paper [22], the authors have emphasized that the error of approximation for both schemes is $O\left(h^{2}+\tau\right)$ and the numerical algorithm for a semi-implicit scheme is very economical and fast. 
In $[8,9,11,12,16,17,23,24,30]$ the conditions of stability of difference schemes for one-dimensional parabolic equations with integral and other types of nonlocal boundary conditions have been obtained. In all papers the investigation of stability was based on a priori estimations, the maximum principle, structure of the spectrum of the transmission matrix or some modifications of these methods.

In our paper, we develop an idea of semi-implicit schemes by using the theory of $M$-matrices. As much as it is known for the authors, application of $M$-matrices in the investigation of the stability of difference schemes for the problems with nonlocal conditions was not considered before.

Application of the $M$-matrices in the convergence of iterative methods of difference problems with nonlocal conditions, arising from elliptic equations have been considered in $[13,36]$.

The structure of this paper is as follows. In Section 2, the semi-implicit scheme for boundary problem (1.1)-(1.3) is formulated and rewritten in the form appropriate for the application of $M$-matrices theory. In Section 3 the stability of the difference scheme is proven. A review on cases, where a stability analysis methodology based on the $M$-matrices theory can be applied is described in Section 4. In Section 5 the results of numerical experiment are provided. Last Section 6 is devoted to some generalizations and conclusions.

\section{Semi-implicit difference scheme}

We define a semi-implicit scheme for the boundary problem (1.1)-(1.3). First of all, we formulate an assumption for the function $K(x, y, \xi, \eta)$.

Assumption 1 For all $(x, y) \in \partial \Omega$

$$
K(x, y, \xi, \eta) \geq 0 .
$$

Assumption $2 K(x, y, \xi, \eta)$ is sufficiently smooth and such that, for any function $v(x, y) \in C_{2}(\bar{\Omega})$, the following numerical integration formula is valid

$$
\iint_{\Omega} K(x, y, \xi, \eta) v(\xi, \eta) d \xi d \eta=h^{2} \sum_{m, l=1}^{N-1} \rho_{m l} K\left(x, y, x_{m}, y_{l}\right) v_{m l}+O\left(h^{2}\right)
$$

for all $(x, y) \in \partial \Omega$.

Now we introduce uniform grids $\Omega_{h}, \bar{\Omega}_{h}, \Gamma_{h}$ and $\omega_{\tau}$ with grid steps $h$ and $\tau$ :

$$
\begin{aligned}
& \Omega_{h}=\left\{\left(x_{i}, y_{j}\right): x_{i}=i h, y_{j}=j h, i, j=\overline{1, N-1} ; h=1 / N\right\}, \\
& \Gamma_{h}=\left\{\left(x_{i}, y_{j}\right): i, j \in\{0, N\}\right\}, \quad \bar{\Omega}_{h}=\Omega_{h} \cup \Gamma_{h}, \\
& \omega_{\tau}=\left\{t^{n}: t^{n}=n \tau, n=\overline{1, M} ; \tau=T / M\right\} .
\end{aligned}
$$

We define the semi-implicit difference scheme

$$
\begin{aligned}
& \partial_{t} u_{i j}^{n}=\delta_{x}^{2} u_{i j}^{n}+\delta_{y}^{2} u_{i j}^{n}-q_{i j} u_{i j}^{n}+f_{i j}^{n}, \quad(i, j) \in \Omega_{h}, \\
& u_{i j}^{n}=K_{i j}\left(\left\{u_{m l}^{n-1}\right\}\right)+\mu_{i j}^{n}, \quad(i, j) \in \Gamma_{h}, \\
& u_{i j}^{0}=\varphi_{i j}, \quad(i, j) \in \bar{\Omega}_{h},
\end{aligned}
$$


where

$$
\begin{aligned}
& \partial_{t} u_{i j}^{n}=\left(u_{i j}^{n}-u_{i j}^{n-1}\right) / \tau \\
& \delta_{x}^{2} u_{i j}=\frac{k_{i-\frac{1}{2}, j} \cdot u_{i-1, j}^{n}-\left(k_{i+\frac{1}{2}, j}+k_{i-\frac{1}{2}, j}\right) u_{i j}^{n}+k_{i+\frac{1}{2}, j} \cdot u_{i+1, j}^{n}}{h^{2}}, \\
& \delta_{y}^{2} u_{i j}=\frac{k_{i, j-\frac{1}{2}} \cdot u_{i, j-1}^{n}-\left(k_{i, j+\frac{1}{2}}+k_{i, j-\frac{1}{2}}\right) u_{i j}^{n}+k_{i, j+\frac{1}{2}} \cdot u_{i, j+1}^{n}}{h^{2}}, \\
& K_{i j}\left(\left\{u_{m l}^{n-1}\right\}\right)=h^{2} \sum_{m, l=1}^{N-1} \rho_{m l} K\left(x_{i}, y_{j}, x_{m}, y_{l}\right) u_{m l}^{n-1} .
\end{aligned}
$$

The weights $\rho_{m l}$ are defined as follows: $\rho_{m l}=\rho_{m} \rho_{l}$,

$$
\rho_{k}=\left\{\begin{array}{l}
1, \quad k=\overline{3, N-3} \\
1 / 2, \quad k=2, N-2 \\
2, \quad k=1, N-1
\end{array}\right.
$$

The differential equation is replaced by the difference scheme (2.2), (2.5), (2.6), as usual [29]. More explicitly we will comment the approximation of integral condition (1.2) by formulas $(2.3),(2.7),(2.8)$. These formulas are obtained in the following way.

In one-dimensional case, let us write

$$
\int_{a}^{b} v(x) d x=\int_{x_{0}}^{x_{2}} v(x) d x+\sum_{i=2}^{N-3} \int_{x_{i}}^{x_{i+1}} v(x) d x+\int_{x_{N-2}}^{x_{N}} v(x) d x
$$

where $\frac{b-a}{N}=h$. We replace approximately the integrals in the partial intervals $\left[x_{i}, x_{i+1}\right]$ according to the trapezoid rule

$$
\int_{x_{i}}^{x_{i+1}} v(x) d x \approx \frac{v_{i}+v_{i+1}}{2} h, \quad i=\overline{2, N-3} .
$$

In the rest of the intervals, we use the central rectangular formula

$$
\int_{x_{0}}^{x_{2}} v(x) d x=v_{1} \cdot 2 h, \quad \int_{x_{N-2}}^{x_{N}} v(x) d x=v_{N-1} \cdot 2 h .
$$

So, we get a new numerical integration formula

$$
\int_{a}^{b} v(x) d x=h \sum_{i=1}^{N-1} \rho_{i} v_{i}+O\left(h^{2}\right)
$$

where

$$
\rho_{i}=\left\{\begin{array}{l}
1, \quad i=\overline{3, N-3} \\
1 / 2, \quad i=2, N-2 \\
2, \quad i=1, N-1
\end{array}\right.
$$


In this formula, only integer indexes $i$ (like in the trapezoid rule) are used and there are no values of the function $v(x)$ at the ends of the interval (like in the rectangular formula). This was precisely our aim for using the $M$-matrices theory. If $v(x) \in C_{2}[a, b]$, then the error of numerical integration formula (2) is $O\left(h^{2}\right)$.

For the function with two variables, it follows from (2)

$$
\iint_{\Omega} v(x, y) d x d y=h^{2} \sum_{m, l=1}^{N-1} \rho_{m l} v_{m l}+O\left(h^{2}\right),
$$

where $\rho_{m l}=\rho_{m} \rho_{l}$.

In this case, the error of approximation of difference problem (2.2)-(2.8) is $O\left(h^{2}+\tau\right)$.

On every layer $t^{n} \in \omega_{\tau}$ there are $(N+1)^{2}$ equations and variables $u_{i j}^{n}$, $(i, j) \in \bar{\Omega}_{h}$ in the system $(2.2)-(2.8)$. We rewrite this system of equations in the matrix form, where the vectors are of the order $(N-1)^{2}$. With this aim we write $u_{i j}^{n}(i, j) \in \Gamma_{h}$ from formula (2.3) into these equations (2.2), in which $i=1, N-1$ or $j=1, N-1$. Then we get

$$
\begin{gathered}
\partial_{t} u_{i j}^{n}=\partial_{x}^{2} u_{i j}^{n}+\partial_{y}^{2} u_{i j}^{n}-q_{i j} u_{i j}^{n}+f_{i j}^{n}, \quad i, j=\overline{2, N-2} \\
\partial_{t} u_{i j}^{n}=\tilde{\delta}_{x}^{2}\left(u_{i j}^{n}, u_{i j}^{n-1}\right)+\tilde{\delta}_{y}^{2}\left(u_{i j}^{n}, u_{i j}^{n-1}\right)-q_{i j} u_{i j}^{n}+\tilde{f}_{i j}^{n}, \\
\quad i=1, N-1 \text { or } j=1, N-1,
\end{gathered}
$$

where

$$
\begin{aligned}
& \tilde{\delta}_{x}^{2}\left(u_{i j}^{n}, u_{i j}^{n-1}\right)=\left\{\begin{array}{c}
h^{-2}\left(k_{i-\frac{1}{2}, j} h^{2} \sum_{m, l=1}^{N-1} \rho_{m l} K\left(x_{0}, y_{j}, x_{m}, y_{l}\right) u_{m l}^{n-1}\right. \\
\left.-\left(k_{i-\frac{1}{2}, j}+k_{i+\frac{1}{2}, j}\right) u_{i j}^{n}+k_{i+\frac{1}{2}, j} u_{i+1, j}^{n}\right), \quad i=1, \\
h^{-2}\left(k_{i-\frac{1}{2}, j} u_{i-1, j}^{n}-\left(k_{i-\frac{1}{2}, j}+k_{i+\frac{1}{2}, j}\right) u_{i j}^{n}\right. \\
\left.+k_{i+\frac{1}{2}, j} h^{2} \sum_{m, l=1}^{N-1} \rho_{m l} K\left(x_{N}, y_{j}, x_{m}, y_{l}\right) u_{m l}^{n-1}\right), i=N-1,
\end{array}\right. \\
& \tilde{\delta}_{y}^{2}\left(u_{i j}^{n}, u_{i j}^{n-1}\right)=\left\{\begin{array}{l}
h^{-2}\left(k_{i, j-\frac{1}{2}} h^{2} \sum_{m, l=1}^{N-1} \rho_{m l} K\left(x_{i}, y_{0}, x_{m}, y_{l}\right) u_{m l}^{n-1}\right. \\
\left.\quad-\left(k_{i, j-\frac{1}{2}}+k_{i, j+\frac{1}{2}}\right) u_{i j}^{n}+k_{i, j+\frac{1}{2}} u_{i, j+1}^{n}\right), \quad j=1, \\
h^{-2}\left(k_{i, j-\frac{1}{2}} u_{i, j-1}^{n}-\left(k_{i, j-\frac{1}{2}}+k_{i, j+\frac{1}{2}}\right) u_{i j}^{n}\right. \\
\left.+k_{i, j+\frac{1}{2}} h^{2} \sum_{m, l=1}^{N-1} \rho_{m l} K\left(x_{i}, y_{N}, x_{m}, y_{l}\right) u_{m l}^{n-1}\right), j=N-1,
\end{array}\right. \\
& \tilde{f}_{i j}^{n}= \begin{cases}f_{i j}^{n}+h^{-2} k_{i-\frac{1}{2}, j} \mu_{i j}^{n-1}, & i=1, \\
f_{i j}^{n}+h^{-2} k_{i+\frac{1}{2}, j} \mu_{i j}^{n-1}, & i=N-1, \\
f_{i j}^{n}+h^{-2} k_{i, j-\frac{1}{2}} \mu_{i j}^{n-1}, & j=1, \\
f_{i j}^{n}+h^{-2} k_{i, j+\frac{1}{2}} \mu_{i j}^{n-1}, & j=N-1 .\end{cases}
\end{aligned}
$$


There are $(N-1)^{2}$ equations and unknowns $u_{i j}^{n}, i, j=\overline{1, N-1}$ in the system of equations (2.10)-(2.11).

The initial system of difference equations on one layer $(2.2),(2.3)$ is equivalent to the newly obtained system (2.10)-(2.11) with formulas (2.3). The main difference of these systems is that it is possible to solve the system (2.10)-(2.11) both in one and in all layers, independently of nonlocal conditions (2.3). In other words in the system of equations (2.10)-(2.11) there are no unknowns $u_{i j}^{n}, u_{i j}^{n-1},(i, j) \in \Gamma_{h}$.

We write difference equation system (2.10)-(2.11) in the matrix form

$$
U^{n}-U^{n-1}=\tau\left(\Lambda U^{n}+C U^{n-1}\right)+\tau \tilde{f}
$$

where $U^{n}=\left\{u_{i j}^{n}\right\},(i, j) \in \Omega_{h}$ and $\tilde{f}$ are the vectors of $(N-1)^{2}$ order, $\Lambda$ and $C$ are square matrices of the same order.

Matrix $\Lambda$ consist of the coefficients of the unknowns $u_{i j}^{n}$ on the right side of system $(2.10),(2.11)$. In other words, $\Lambda$ is matrix of the difference equations with a Dirichlet boundary condition.

Analogously, matrix $C$ is formed from the coefficients of the unknowns $u_{i j}^{n-1}$ of equations (2.11), i.e. matrix $C$ consist of the coefficients of nonlocal condition $(2.3)$.

Now we write a system of equations (2.12) as a two-layer difference scheme

$$
(I-\tau \Lambda) U^{n}=(I+\tau C) U^{n-1}+\tau \tilde{f}
$$

or

$$
U^{n}=S U^{n-1}+g
$$

where

$$
S=(I-\tau \Lambda)^{-1}(I+\tau C), \quad g=(I-\tau \Lambda)^{-1} \tau \tilde{f} .
$$

Remark 1. Let us set down the Assumptions 1 and 2 and, moreover, there exists a constant $0<\gamma<1$ that

$$
\iint_{\Omega} K(x, y, \xi, \eta) d \xi d \eta \leq \gamma<1, \quad(x, y) \in \partial \Omega .
$$

So it is possible to choose sufficiently small $h_{0}$, such that, for all $0<h \leq h_{0}$, the inequality

$$
h^{2} \sum_{m, l=1}^{N-1} \rho_{m l} K\left(x_{i}, y_{j}, x_{m}, y_{l}\right) \leq \rho^{*}<1, \quad\left(x_{i}, y_{j}\right) \in \Gamma_{h}
$$

would be correct.

However, our proof of stability is not based on inequality (2.14). We reserved this assumption only there, where we want to compare the results, which we have obtained with the results of the paper [22] (see Case 1 in the Section 4). 
Remark 2. The order of system of difference equations (2.2)-(2.3) written on a single layer, is $(N+1)^{2}$. However, aiming to write it in the matrix form (2.9) or $(2.13)$, we firstly reduce it to a system of the order $(N-1)^{2}$. In other words, we divided system $(2.2)-(2.3)$ into two parts: the system of the order $(N-1)^{2}$ with the unknowns $u_{i j}^{n}, i, j=1,2, \ldots, N-1$, only at the interval points of the area $\Omega_{h}$ and separately explicit formulas (2.3) for the unknowns at the contour points $\Gamma_{h}$. This is related to the methodology of application of $M$-matrices theory, described in Section 4. The matter is that the eigenvalue problem

$$
\begin{aligned}
& \delta_{x}^{2} u_{i j}+\delta_{y}^{2} u_{i j}-q_{i j} u_{i j}+\lambda u_{i j}=0, \quad(i, j) \in \Omega_{h}, \\
& u_{i j}=K_{i j}\left(\left\{u_{i j}\right\}\right), \quad(i, j) \in \Gamma_{h}
\end{aligned}
$$

corresponding to system (2.2)-(2.3) of difference equations on a single layer, possesses not $(N+1)^{2}$, but $(N-1)^{2}$ eigenvalues, i.e. it is equivalent to the algebraic eigenvalue problem with the matrix of the order $(N-1)^{2}$ (more in detail see $[30,35])$, while in the theory of $M$-matrices the role of eigenvalues is very important.

Remark 2 allows us to explain why, when approximating differential problem (1.1)-(1.3) by semi-implicit difference scheme (2.2)-(2.4), we use not the classical but new type formula of numerical integration (2.7). Let us take any other standard formula of numerical integration instead of formula (2.7), for example, the trapezoid formula

$$
K_{i j}\left(\left\{u_{m l}^{n-1}\right\}\right)=h^{2} \sum_{m . l=0}^{N} K\left(x_{i}, y_{j}, x_{m}, y_{l}\right) u_{m l}^{n-1},
$$

in which $\rho_{m l}$ are weight coefficients. Let us now insert the values of $u_{i j}^{n},(i, j) \in$ $\Gamma_{h}$ from formula (2.16) into that equations of (2.2), in which $i=1, N-1$ or $j=1, N-1$. We get a system of equations, analogous to system equations to system (2.10)-(2.11), in which the coordinates of the vector $u^{n}$ of the order $(N-1)^{2}$ are connected with the coordinates of the vector $u^{n-1}$ of the order $(N+1)^{2}$. It is not possible to write such a system in the matrix form, analogous to $(2.12)$ with a square matrix.

\section{Stability of a difference scheme}

We investigate the stability of semi-implicit difference scheme (2.13). We use the following definition of stability.

Definition 1. Difference scheme (2.13) is stable with respect to the initial data, if

$$
\rho(S)=\max |\lambda(S)|<1 .
$$

One of the first papers where such a definition of stability was used is [4]. In that paper the authors call, this kind of stability as a stepwise stability. The ground for such a definition of stability is permitted, because even for non-symmetrical matrices it is possible to define norm [2]:

$$
\|S\|_{*}=\rho(S) .
$$


Referring to such a definition of stability, in papers $[4,14,15,30,32]$, the stability of difference schemes with a variety of nonlocal conditions was investigated. An important feature of the condition (3.1) is that the stability is proven in certain, sometimes even complicated, energetic norms of vectors. In some cases the equivalence of such norms to some simpler norms, for example $L_{2}$-norm, is proven $[16,17]$.

If matrix $S$ is of simple structure, then the norm of a vector compatible with the matrix norm $\|S\|_{*}$, could be defined in the following way $[15,19,30]$ :

$$
\|u\|_{*}=\left(P^{-1} u, P^{-1} u\right)^{\frac{1}{2}}
$$

where $P$ is a matrix, the columns of which are linear independent eigenvectors of matrix $S$.

In general, when $S$ is not obligatorily the matrix of simple structure, the compatible norm of the vector is defined in a more complicated way (see, e.g., [2], Ch. 7.3, or [28], Ch. II.2, § 3.4). The important property of norm (3.2) is that in the case $\rho(S)>1$, the difference scheme is not stable in any vectorial norm $[4,17,19]$.

Now we remind the definition of an $M$-matrix.

Definition 2. The square matrix $A=\left\{a_{i j}\right\}, i, j=\overline{1, n}$ is called an $M$-matrix, if it is non-singular, $a_{i j} \leq 0$, as $i \neq j$ and all the elements of matrix $A^{-1}$ are nonnegative (we denote it as $A^{-1} \geq 0$ ).

As a conclusion from this definition, it follows that $a_{i i}>0$.

Lemma 1. Matrix $(-\Lambda)$ is an M-matrix.

Proof. The statement of lemma is a well-known statement, only it is wording of unusual form. Indeed, as mentioned above, $\Lambda$ is the matrix of the system of difference equations (2.2) with the Dirichlet condition. So, the diagonal elements of the matrix $-\Lambda$ are positive, non-diagonal - non-positive. The matrix is diagonally dominant in a weak sense and irreducible. It is enough of these four properties for matrix $-\Lambda$ to be an $M$-matrix $[3,37]$.

Lemma 2. If Assumption 1 is true, then $C \geq 0$.

Proof. Indeed matrix $C$, as mentioned above is composed of the coefficients to unknowns $u_{i j}^{n-1}$ of equations (2.11), i.e. $C \geq 0$.

Let us take now the matrix $-(\Lambda+C)$. For this matrix the following necessary $M$-matrix condition is valid: the diagonal elements of this matrix are positive, and non-diagonal ones are non-positive according to Lemmas 1 and 2. But this is not enough for this matrix to be an $M$-matrix. Then, at first we set up an assumption.

Assumption 3 Matrix $-(\Lambda+C)$ is an $M$-matrix. 
In Section 5 we discuss more exhaustively when this assumption is correct. Now we describe the methodology, how the theory of $M$-matrices could be applied to investigate of the stability of difference schemes.

Now, to prove the stability of the difference scheme, we use the notion of regular splitting.

Definition 3. Let $A$ be an $M$-matrix. The representation $A=M-N$, where $M^{-1} \geq 0, N \geq 0$ is called the regular splitting of matrix $A$.

Theorem 1. If the Assumptions 1 and 3 are satisfied, then difference scheme (2.13) is stable with respect to the initial data.

Proof. Let us consider matrix $-(\Lambda+C)$ and then set down the identity

$$
-\tau(\Lambda+C)=I-\tau \Lambda-(I+\tau C)
$$

If $-\Lambda$ is an $M$-matrix, then $-\tau \Lambda$ is also an $M$-matrix. Further increasing the diagonal elements of an $M$-matrix, the newly obtained matrix is also an $M$-matrix [3,37]. Thus, $I-\tau \Lambda$ is an $M$-matrix, and therefore $(I-\tau \Lambda)^{-1} \geq 0$. Further, if $C \geq 0$, then $I+\tau C \geq 0$. So, splitting (3.3) is a regular splitting of matrix $-\tau(\Lambda+C)$. According to one of the main properties of regular splitting $[3,37]$,

$$
\rho(S)=\rho\left((I-\tau \lambda)^{-1}(I+\tau C)\right)<1 .
$$

The theorem is proved.

\section{Cases, where $-(\Lambda+C)$ is an $M$-matrix}

In this Section, we provide already known as well as new results, whenever it is possible to apply the theory of $M$-matrices (Theorem 1 ) in the investigation of the stability of difference schemes. In other words, we will consider concrete cases, where Assumption 3 is valid.

Case 1. Let us assume that inequality (2.14) is valid for the given function $K(x, y, \xi, \eta) \geq 0$. Then, according to Remark 1 inequality (2.15) is correct. As mentioned in Section 3, the diagonal elements of the matrix $-(\Lambda+C)$ are positive and non-diagonal are non-positive. Taking into account inequality (2.15), we directly verify that the matrix $-(\Lambda+C)$ is diagonally dominant in week sense. Further on, the matrix $-(\Lambda+C)$ is an irreducible one. Indeed, this matrix is obtained from the irreducible matrix $-\Lambda$, replacing some zero elements by nonzero ones. Therefore, it is also irreducible. From these four properties of the matrix $-(\Lambda+C)$ it follows that it is an $M$-matrix.

However, if condition (2.14) is correct, then the stability of the difference scheme can be to proved by other methods, as well for example, based on the maximum principle. Such a proof is presented in [22] under the conditions $k(x, y)=1, q(x, y)=f(x, y, t)=\mu(x, y, t)=0$. In this particular case it is proven, that solution of semi-implicit scheme has the property

$$
0<u^{n}<u^{n-1}
$$


where $u^{n}=\max _{i, j}\left|u_{i j}^{n}\right|$. If the difference scheme has this property, then the solution of difference as well as differential problem describes only the decreasing diffusion processes. It is quite strict limitation for the solving a differential problem. We note that, such limitation is connected only with assumption $q=f=\mu=0$, but not with the maximum principle. The problems we are considering and the results obtained have no limitations of this type (see also Section 5).

Case 2. Let us take $K(x, y, \xi, \eta)=\gamma=$ const. Assumption 1 and inequality (2.14) in this case mean that $0 \leq \gamma|\Omega|<1$. Where $|\Omega|$ is the area of the square $\Omega$, as $\gamma|\Omega| \geq 1$, inequality (2.15) is not correct and it is not possible to use maximum principle for the investigating of the stability. In order to apply the $M$-matrices theory in the case of $\gamma|\Omega| \geq 1$, we use the results of paper [38] about the eigenvalues of the differential operator. From the results of [38] it follows that all the eigenvalues of the eigenvalue problem

$$
\begin{aligned}
& \frac{\partial}{\partial x}\left(k(x, y) \frac{\partial u}{\partial x}\right)+\frac{\partial}{\partial y}\left(k(x, y) \frac{\partial u}{\partial y}\right)-q u+\lambda u=0, \quad(x, y) \in \Omega, \\
& u(x, y)=\gamma \iint_{\Omega} u(\xi, \eta) d \xi d \eta, \quad(x, y) \in \partial \Omega
\end{aligned}
$$

if $q=0$ are positive $(\lambda>0)$, if the condition

$$
0 \leq \gamma<1 /|\Omega|
$$

is fulfilled. If $\gamma=1 /|\Omega|$, then $\lambda=0$ is an eigenvalue, while a negative eigenvalue exists only in case $\gamma>1 /|\Omega|$. When $q>0$ in equation (4.1), then eigenvalues of eigenvalue problem (4.1), (4.2) can increase. So, all the eigenvalues, depending on $q$, can be positive even under the condition $\gamma>|\Omega|$.

In the one-dimensional case, it is possible to determine the relation between $q$ and $\varphi$, in the presence of which the inequality $\lambda>0$ is true. Namely, it follows from [20] that all eigenvalues of the eigenvalue problem

$$
\begin{aligned}
& \frac{d^{2} u}{d x^{2}}-q u+\lambda u=0, \quad q=\text { const }>0, \quad x \in(0,1), \\
& u(0)=\gamma_{1} \int_{0}^{1} u(x) d x, \quad u(1)=\gamma_{2} \int_{0}^{1} u(x) d x
\end{aligned}
$$

are positive, if

$$
0<\gamma_{1}+\gamma_{2}<\sqrt{q} / \tanh (\sqrt{q} / 2) .
$$

We note that, under the condition $q>0$ the function

$$
\varphi(q)=\sqrt{q} / \tanh (\sqrt{q} / 2)
$$

is monotonically decreasing and $\varphi(q)>2$.

We have indicated the properties of the spectrum of a differential operator with the nonlocal condition. However, as noticed in [21], similar results are valid for discrete approximation of the diffusion operator (see also [18, 26, 35] and references therein). 
So properly selecting $a$ and $q(x, y)$, the matrix $-(\Lambda+C)$ in equation $(2.12)$ can remain an $M$-matrix, as $\gamma>1$. Indeed, the diagonal elements of the matrix $-(\Lambda+C)$ are positive, nondiagonal are nonpositive and all the eigenvalues are positive. So, the matrix is an $M$-matrix. But this matrix is not diagonally dominant, therefore we cannot investigate the stability based on the maximum principle. More exhaustively it is described in Section 5. The spectrum of the differential and difference operators with the variable coefficient has been investigated in $[27,33,35]$.

Case 3. Another example is related to a two-dimensional parabolic equation (1.1) and a little bit different integral condition. Let us take, for example, the following boundary conditions

$$
\begin{aligned}
& u(0, y, t)=\gamma_{1} \int_{0}^{a} u(x, y, t) d x+\mu_{1}(y, t), \\
& u(a, y, t)=\gamma_{2} \int_{0}^{a} u(x, y, t) d x+\mu_{2}(y, t), \\
& u(x, 0, t)=\mu_{3}(x, t), \quad u(x, a, t)=\mu_{4}(x, t) .
\end{aligned}
$$

When $k(x, y)=1, a=1$, it follows from the results in [36], that $-(\Lambda+C)$ is an $M$-matrix, if $0<\gamma_{1}+\gamma_{2}<\gamma_{*} \approx 3.42$. The results with the variable coefficient $k(x, y)$ are summarized in [35].

\section{$5 \quad$ Numerical results}

In this section, we present some results of numerical experiment. As a test problem we solve the following two-dimensional linear parabolic equation with a nonlocal boundary condition

$$
\begin{aligned}
& \frac{\partial u}{\partial t}=\frac{\partial^{2} u}{\partial x^{2}}+\frac{\partial^{2} u}{\partial y^{2}}-q(x, y) u+f(x, y, t), \quad(x, y) \in \Omega=\{0<x, y<a\} \\
& u(x, y, t)=\gamma \iint_{\Omega} u(x, y, t) d x d y+\mu(x, y, t), \quad(x, y) \in \partial \Omega \\
& u(x, y, 0)=\varphi(x, y), \quad(x, y) \in \bar{\Omega} .
\end{aligned}
$$

This differential problem is approximated by difference scheme (2.2)-(2.8).

One of the aims of the numerical experiment is to illustrate more exhaustively the necessity of condition (4.3) in the case $q(x, y)=0$. The second aim is to investigate the influence of function $q(x, y)$ on the stability of difference scheme.

The functions $f(x, y, t), \mu(x, y, t)$, and $\varphi(x, y)$ were chosen so that the solution of the problem would be the increasing function of argument $t$ :

$$
u(x, y, t)=\sin (\pi x) \sin (\pi y) e^{t} .
$$

In every layer $t^{n}$, the system of difference equations (2.2)-(2.8) was solved by Jacobi method. In other words, trying to obtain $u_{i j}^{n}$, when the values $u_{i j}^{n-1}$, 
$(i, j) \in \bar{\Omega}_{h}$ are known, we solve the system of difference equations (2.2) with the boundary conditions of Dirichlet type (2.3) by simple iterative method.

In Table 1, the results of solution of system (2.2)-(2.3) are provided for different values of $\gamma, h$ and $\tau$, as $q(x, y)=0, a=1, T=1$. The values of error

$$
\varepsilon=\max _{i j}\left|u_{i j}^{n}-u_{i j}^{*}\right|,
$$

where $u_{i j}^{*}$ is the exact solution $u\left(x_{i}, y_{j}\right)$ of the differential problem, are presented in this table. It follows from the results, that errors change precisely enough according to the law $O\left({ }^{2}+\tau\right)$, when the condition of stability (4.3) is fulfilled. When $\gamma=1$, the error slightly deviates from this low when $\gamma$ is growing $(\gamma>1)$, the difference scheme becomes unstable.

Table 1. The errors of solution in the case $q(x, y)=0, a=1, T=1$; stability condition (4.3) is $\gamma<1$.

\begin{tabular}{lllll}
\hline$h$ & $\frac{1}{20}$ & $\frac{1}{40}$ & $\frac{1}{80}$ & $\frac{1}{160}$ \\
\hline$\tau$ & $\frac{1}{40}$ & $\frac{1}{160}$ & $\frac{1}{640}$ & $\frac{1}{2560}$ \\
\hline$\gamma=0$ & 0.0070 & 0.0017 & 0.0004 & 0.0001 \\
$\gamma=0.5$ & 0.0264 & 0.0070 & 0.0018 & 0.0004 \\
$\gamma=0.9$ & 0.1570 & 0.0467 & 0.0122 & 0.0031 \\
$\gamma=1$ & 0.3199 & 0.1194 & 0.0339 & 0.0088 \\
$\gamma=1.1$ & 0.8016 & 0.5093 & 0.1856 & 0.0522 \\
$\gamma=1.2$ & 2.4494 & 3.6792 & 2.0831 & 0.6735 \\
\hline
\end{tabular}

Tables 2 and 3 are composed analogously for two other values of $a$.

Table 2. The errors of solution in the case $q(x, y)=0, a=0.5, T=1$; stability condition (4.3) is $\gamma<4$.

\begin{tabular}{lllll}
\hline$h$ & $\frac{1}{20}$ & $\frac{1}{40}$ & $\frac{1}{80}$ & $\frac{1}{160}$ \\
\hline$\tau$ & $\frac{1}{40}$ & $\frac{1}{160}$ & $\frac{1}{640}$ & $\frac{1}{2560}$ \\
\hline$\gamma=0$ & 0.0013 & 0.00032 & 0.00008 & 0.00002 \\
$\gamma=1$ & 0.0086 & 0.0024 & 0.0006 & 0.00016 \\
$\gamma=3.9$ & 0.3697 & 0.1678 & 0.0517 & 0.0152 \\
$\gamma=4$ & 0.5032 & 0.3125 & 0.1194 & 0.0386 \\
$\gamma=4.1$ & 0.7113 & 0.7223 & 0.4393 & 0.1774 \\
$\gamma=4.2$ & 1.0440 & 2.0776 & 2.5458 & 1.4185 \\
\hline
\end{tabular}

When studying the results presented in Table 1 - Table 3 , there may rise an impression that the condition for the stability $\gamma<\frac{1}{|\Omega|}$ is not necessary. Then, stability remains, in particular case $a=1$, at least for the values $\gamma=1.1$ or $\gamma=1.2$. However, as noted in [34], in the case $T=1$, it is not always possible to draw proper conclusions from the numerical results on the limits of stability and instability regions. It is because the estimation of stability usually includes the multiplier $e^{-\lambda t}$, where $\lambda$ is an eigenvalue of the matrix $-(\Lambda+C)$. When 
Table 3. The errors of solution in the case $q(x, y)=0, a=1.5, T=1$; stability condition (4.3) is $\gamma<0.444$.

\begin{tabular}{lllll}
\hline$h$ & $\frac{1}{20}$ & $\frac{1}{40}$ & $\frac{1}{80}$ & $\frac{1}{160}$ \\
\hline$\tau$ & $\frac{1}{40}$ & $\frac{1}{160}$ & $\frac{1}{640}$ & $\frac{1}{2560}$ \\
\hline$\gamma=0$ & 0.0076 & 0.0019 & 0.0005 & 0.0001 \\
$\gamma=0.4$ & 0.0123 & 0.0032 & 0.0009 & 0.0013 \\
$\gamma=0.444$ & 0.0192 & 0.0055 & 0.0017 & 0.0019 \\
$\gamma=0.5$ & 0.0401 & 0.0145 & 0.0050 & 0.0051 \\
$\gamma=0.55$ & 0.0899 & 0.0442 & 0.0169 & 0.0174 \\
$\gamma=0.6$ & 0.2222 & 0.1667 & 0.0745 & 0.0757 \\
\hline
\end{tabular}

the negative eigenvalue appears, even comparably small in absolute quantity, the multiplier $e^{-\lambda t}$, depending on $T$, may become high and always increases unlimitedly, when $T$ is growing. This fact causes instability.

In Table 4 , the values of error $\varepsilon_{1}$

$$
\varepsilon_{1}=\frac{\max _{i, j}\left|u_{i j}^{n}-u_{i j}^{*}\right|}{\max _{i, j}\left|u_{i j}^{*}\right|} \leq \max _{i, j} \frac{\left|u_{i j}^{n}-u_{i j}^{*}\right|}{\left|u_{i j}^{*}\right|}=\varepsilon_{r}
$$

are presented for different values of $T$, where $\varepsilon_{r}$ is a relative error.

Table 4. The relative error $\varepsilon_{1}$ in the case $q(x, y)=0, a=1, h=\frac{1}{80}, \tau=\frac{1}{640}$

\begin{tabular}{lllll}
\hline$T$ & 1 & 2 & 5 & 10 \\
\hline$\gamma=1$ & 0.0130 & 0.0171 & 0.0191 & 0.0192 \\
$\gamma=1.05$ & 0.0276 & 0.0659 & 0.3252 & 2.5404 \\
$\gamma=1.1$ & 0.0714 & 0.5006 & 118.6338 & $1.0355 \cdot 10^{6}$ \\
\hline
\end{tabular}

The absolute error $\varepsilon$, as it is evident, may grows because of unlimited growth of the solution of the differential problem, even in the case of stability. To demonstrate the instability it is difficult to use the relative error $\varepsilon_{r}$ because the solution $u_{i j}^{*}$ is equal to zero at the same points of $\bar{\Omega}_{h}$. Thus, we can use the error $\varepsilon_{1}$ for demonstrating the instability in case $\gamma>1$ : if $\varepsilon_{1}$ grows, then the relative error $\varepsilon_{r}$ grows as well.

From the numerical results presented in Table 4 we may expect that the sufficient condition of stability, formulated in inequality (4.3), at least in the case $K=$ const and $a=1$, is close to the necessary condition or corresponds to it precisely.

Results of the numerical experiment presented in Table 5 shows the influence of the coefficient $q(x, y)$ for the stability of difference scheme. As it was mentioned in Section 4, matrix $-(\Lambda+C)$ remains an $M$-matrix, when coefficients $q(x, y)$ is increasing. It follows from the numerical results, that increasing the coefficients $q(x, y)$, the properties of matrix $-(\Lambda+C)$ becomes better. In other words, the area of the stability of difference scheme is increasing - we 
Table 5. The error of solution in the case $q(x, y)=q(1+c x)(1+c y), h=1 / 160$, $\tau=1 / 2560, T=1, a=1$

\begin{tabular}{lllll}
\hline$q$ & $q=0$ & $q=5$ & $q=10$ & $q=10$ \\
\hline$c$ & $c=0$ & $c=0$ & $c=0$ & $c=1$ \\
\hline$\gamma=0$ & 0.00046 & 0.00037 & 0.00031 & 0.00022 \\
$\gamma=1$ & 0.0146 & 0.0040 & 0.0024 & 0.0015 \\
$\gamma=1.2$ & 1.1598 & 0.0375 & 0.0072 & 0.0026 \\
$\gamma=1.5$ & 30474 & 323.4655 & 4.2877 & 0.0103 \\
$\gamma=1.7$ & $1.1747 \cdot 10^{8}$ & $1.1019 \cdot 10^{6}$ & 11118 & 0.4015 \\
$\gamma=2$ & $2.0417 \cdot 10^{14}$ & $1.8018 \cdot 10^{12}$ & $1.6367 \cdot 10^{10}$ & $2.0788 \cdot 10^{5}$ \\
\hline
\end{tabular}

observe stability of the scheme with lager values of $\gamma$. However, the values of $\gamma$, with which matrix $-(\Lambda+C)$ remains an $M$-matrix and difference scheme remains stable, grows slowly enough, when increasing $q(x, y)$.

\section{Conclusions and generalization}

The application of $M$-matrices in the investigation of stability of difference schemes has a few advantages suitable for problems with nonlocal conditions. First of all, it is not important whether matrix $S$ in the difference scheme (2.13) is symmetric or not. We note that in the presence of nonlocal boundary conditions, the matrix of the system of difference equations is non-symmetric, except very rare cases. Secondly, in the methodology of application of $M$ matrices it is not important whether the coefficients in the differential equation and the nonlocal condition constant or variable.

And thirdly, this methodology equally suits for one-dimensional and twodimensional, and in general $n$-dimensional cases. One of the shortages in this methodology is the condition $K(x, y, \xi, \eta) \geq 0$.

The replacement of the integral condition by the numerical integration formula $(2.3),(2.7)$ plays an important role in the methodology of application of $M$-matrices in investigation of stability of difference schemes. As mentioned above the essence of this formula is that an approximate value of the integral is expressed only by the values of the integral function at the interior (not boundary) points. This fact is related with method of proving and then the algorithm becomes economical.

One of the main conclusions of this paper is that the semi-implicit schemes for the problems with nonlocal conditions are also significant as fully-implicit or explicit difference schemes and have their own merits.

\section{Acknowledgement}

The authors want to thank Prof. Raimondas Čiegis for his suggestions and improvements on the presentation of this article. 


\section{References}

[1] A. Ashyralyev, I.Karatay and P.E. Sobolevskii. On well-posedness of the nonlocal boundary value problem for parabolic difference equations. Discrete Dyn. Nat. Soc., 2:273-286, 2004.

[2] K. E. Atkinson. An Introduction in Numerical Analysis. John Wiley and Sons, 1989.

[3] A. Berman and R. J. Plemmons. Nonnegative Matrices in the Mathematical Sciences. SIAM, Philadelphia, 1994.

[4] B. Cahlon, D.M. Kulkarni and P. Shi. Stepwise stability for the heat equation with a nonlocal constrain. SIAM J. Numer. Anal., 32(2):571-593, 1995. https://doi.org/10.1137/0732025.

[5] J.R. Cannon, Y. Lin and A.L. Matheson. The solution of the diffusion equation in two space variables subject to specification of mass. Applicable Analysis, 50(1-2):1-15, 1993. https://doi.org/10.1080/00036819308840181.

[6] R. Čiegis. Economical difference schemes for the solution of a two-dimensional parabollic problem with an integral condition. Diff Equat, 41(7):1025-1029, 2005. https://doi.org/10.1007/s10625-005-0244-9. Translated from Differentsial'nye Uravneniya, Vol. 41, No. 7, 2005, pp. 975-979.

[7] R. Čiegis, O. Suboč and A.Bugajev. Parallel algorithms for threedimensional parabolic and pseudoparabolic problems with different boundary conditions. Nonl. Anal. Model. Contr., 19(3):382-395, 2014. https://doi.org/10.15388/NA.2014.3.5.

[8] R. Čiegis and N. Tumanova. Numerical solution of parabolic problems with nonlocal boundary conditions. Numer. Funct. Anal. Optim., 31(12):1318-1329, 2010. https://doi.org/10.1080/01630563.2010.526734.

[9] R. Čiegis, A. Štikonas, O. Štikonienè and O. Suboč. A monotonic finite-difference scheme for a parabolic problem with nonlocal condition. Differential Equations, 38(7):1027-1037, 2002. https://doi.org/10.1023/A:1021167932414.

[10] M. Dehghan. Efficient techniques for the second-order parabolic eqution subject to nonlocal specifications. Applied Numerical Mathematics, 52:39-62, 2005. https://doi.org/10.1016/j.apnum.2004.02.002.

[11] G. Ekolin. Finite-difference methods for a nonlocal boundary-value prolbem for head equation. BIT Numerical Mathematics, 31(2):245-261, 1991. https://doi.org/10.1007/BF01931285.

[12] G. Fairweather and J.C. Lopez-Marcos. Galerkin methods for a semilinear parabolic problem with nonlocal conditions. Adv Comput Math, 6(1):243-262, 1996. https://doi.org/10.1007/BF02127706.

[13] V. Griškonienè, M. Sapagovas and O. Štikonienè. Application of M-matrices for solution of a nonlinear eliptic equation with an integral condition. Nonlin. Anal. Model. Contr., 22(4), 2017.

[14] A. V. Gulin. On the spectral stability in subspaces for difference schemes with nonlocal boundary conditions. Diff Equat, 49(7):815-823, 2013. https://doi.org/10.1134/S0012266113070045. Differentsialnye Uravneniya, 2013, Vol. 49, No. 7, pp. 844-852.

[15] A.V. Gulin, N.I. Ionkin and V.A. Morozova. Stability of a nonlocal twodimensional finite-difference problem. Differential Equations, 37(7):970-978, 2001. https://doi.org/10.1023/A:1011961822115. 
[16] A.V. Gulin, N.I. Ionkin and V.A. Morozova. Study of the norm in stability problems for nonlocal difference schemes. Diff Equat, 42(7):974-984, 2006. https://doi.org/10.1134/S0012266106070068. Differentsialnye Uravneniya, 2006, Vol. 42, No. 7, pp. 914-923.

[17] A.V. Gulin, V.A. Morozova and N.S. Udovichenko. Stability of a nonlocal difference problem with a complex parameter. Diff Equat, 47(8):1116-1129, 2011. https://doi.org/10.1134/S0012266111080064.

[18] F. Ivanauskas, T. Meškauskas and M. Sapagovas. Stability of difference schemes for two-dimensional parabolic equations with nonlocal conditions. Appl. Math. Comp, 215(7):2716-2732, 2009. https://doi.org/10.1016/j.amc.2009.09.012.

[19] F. Ivanauskas, Yu. A. Novitski and M. P. Sapagovas. On the stability of an explicit difference scheme for hyperbolic equations with nonlocal boundary conditions. Diff Equat, 49(7):849-856, 2013. https://doi.org/10.1134/S0012266113070070. Differentsialnye Uravneniya, 2013, Vol. 49, No. 7, pp. 877-884.

[20] K. Jakubèlienè and M. Sapagovas. On the stability of a difference scheme for a two-dimensional parabolic equation with an integral condition. Lith. Math. J., 53(3):311-323, 2013. https://doi.org/10.1007/s10986-013-9210-z.

[21] T. Leonavičienè, A. Bugajev, G. Jankevičiūtè and R. Čiegis. On stability analysis of finite difference schemes for generalized Kuramoto-Tsuzuki equation with nonlocal boundary conditions. Math. Model. Anal., 21(5):630-643, 2016. https://doi.org/10.3846/13926292.2016.1198836.

[22] Y. Lin, S. Xu and H. M. Yin. Finite difference approximations for a class of non-local parabolic equations. Intern. J. Math. and Mat. Sci., 20(1):147-163, 1997. https://doi.org/10.1155/S0161171297000215.

[23] J. Martin-Vaquero, A. Queiruga-Dios and A.H. Evicinas. Numerical algorithms for diffusion-reaction problems with non-classical conditions. Appl. Math. Compt., 218(9):5487-5492, 2012. https://doi.org/10.1016/j.amc.2011.11.037.

[24] J. Martin-Vaquero and J. Vigo-Aguiar. On the numerical solution of the heat conduction equations subject to nonlocal conditions. Apl. Numer. Math., 59(10):2507-2514, 2009. https://doi.org/10.1016/j.apnum.2009.05.007.

[25] B.J. Noye and M. Dehgham. New explicit finite difference schemes for twodimensional diffusion subject specification of mass. Numer. Methods Partial Differential Eq., 15:521-534, 1999. https://doi.org/10.1002/(SICI)10982426(199907)15:4;521::AID-NUM7 ¿3.0.CO;2-R.

[26] S. Pečiulytè, O. Štikonienè and A. Štikonas. Sturm - Liouville problem for stationary differential operator with nonlocal integral boundary condition. Math. Model. Anal., 10(4):377-392, 2005. https://doi.org/10.1080/13926292.2005.9637295.

[27] S. Sajavičius and M. Sapagovas. Numerical analysis of the eigenvalue problem for one-dimensional differential operator with nonlocal integral conditions. Nonlin. Anal. Model. Contr., 14(1):115-122, 2009.

[28] A. A. Samarskii and A. V. Gulin. Numerical Methods. Moscow, Nauka, 1989. (in Russian)

[29] A.A. Samarskii. The Theory of Difference Schemes. Moscow, Nauka, 1977 (in Russian); Marcel Dekker, Inc., New York and Basel, 2001. 
[30] M. Sapagovas. On the stability of a finite-difference scheme for nonlocal parabolic boundary-value problems. Lith. Math. J., 48(3):339-356, 2008. https://doi.org/10.1007/s10986-008-9017-5.

[31] M. Sapagovas and K. Jakubèlienè. Alternating direction method for twodimensional parabolic equation with nonlocal integral condition. Nonlin. Anal. Model. Contr., 17(1):91-98, 2012.

[32] M. Sapagovas, G. Kairytè, O. Štikonienè and A. Štikonas. Alternating direction method for a two-dimensional parabolic equation with a nonlocal boundary condition. Math. Model. Anal., 12(1):131-142, 2007. https://doi.org/10.3846/13926292.2007.12.131-142.

[33] M. Sapagovas, R. Čiupaila and Ž. Jokšienè. The eigenvalue problem for a onedimensional differential operator with a variable coefficient and nonlocal integral conditions. Lith. Math. J., 54(3):345-355, 2014. https://doi.org/10.1007/s10986014-9247-7.

[34] M. Sapagovas, R. Čiupaila, Ž. Jokšienė and T. Meškauskas. Computational experiment for stability analysis of difference schemes with nonlocal conditions. Informatica, 24(2):275-290, 2013.

[35] M. Sapagovas, O. Štikonienè, R. Čiupaila and Ž. Jokšienè. Convergence of iterative methods for elliptic equations with integral boundary conditions. Electr. J. Diff. Equat, 2016(118):1-14, 2016.

[36] O. Štikonienè, M. Sapagovas and R. Čiupaila. On iterative methods for some elliptic equations with nonlocal conditions. Nonlin. Anal. Model. Contr., 19(3):517-535, 2014. https://doi.org/10.15388/NA.2014.3.14.

[37] V. V. Voevodin and Y. A. Kuznecow. Matrices and Computations. Moscow, Nauka, 1984. (in Russian)

[38] Y. Wang. Solutions to nonlinear elliptic equations with a nonlocal boundary condition. Electr. J. Diff. Equat, 2002(05):1-16, 2002. 